\title{
Ecotourisme et aires protégées: L'histoire, un potentiel latent pour le complexe OKM (Togo, Afrique)
}

\author{
Dabitora Koumantiga* Kperkouma Wala** \\ Sêmihinva Akpavi**** Badabaté Diwediga***** \\ Komlan Batawila***** Koffi Akpagana******* \\ Universidad de Lomé (Togo)
}

\begin{abstract}
Résumé: Les offres touristiques dans les aires protégées sont le plus souvent orientées vers les potentialités biologiques. Prenant le cas des aires protégées du complexe Oti-Kéran-Mandouri, cette étude relève qu'il existe également un potentiel écotouristique lié à l'histoire à valoriser. Ainsi sur la base d'enquêtes ethnographiques et de visites de terrains, 52 sites historiques regroupant les grottes ancestrales, les reliques coloniales et autres sites liés à l'histoire des peuples ont été identifiés. Ce potentiel écotouristique lié à l'histoire reste latent et sous exploité dans les stratégies de conservation de la biodiversité.
\end{abstract}

Mots Clés: Aires protégées; Ecotourisme; Histoire; Conservation Oti-Kéran-Mandouri; Togoa.

Ecotourism in areas under protection. History as latent potential for the OKM complex in Togo, Africa

Abstract: The tourism on offer in protected areas of Africa is almost invariably directed at discovering the biological potential of the sites. In this case, the Oti-Keran-Mandouri complex is analysed from its potential as a historical site capable of offering ecotourism. Using ethnographic surveys, indivisual interviews and on-site fieldwork, a total of 52 historical sites comprehending ancestral caves, relics of Colonial times and other places contextually important in the history of the people were identified, proving that there is an enormous latent ecotourism potential.

Keywords: Protected Areas; Ecotourism; History; Conservation Oti-Keran-Mandouri; Togo.

\section{Introduction}

La gestion durable des ressources naturelles dans les aires protégées implique des connaissances pluridisciplinaires. Ainsi dans la plupart des pays en développement, les stratégies de gestion efficace des aires protégées doivent intégrer les aspects environnementaux, socioéconomiques, culturels, juridiques et historiques. Busquet (2006) estime que l'intégration de ces différents éléments est un enjeu majeur qui appelle à une approche transdisciplinaire, des sciences sociales aux naturelles et de la théorie à la pratique.

En Afrique, les travaux de recherche (Wala et al., 2012) et les rapports des organisations internationales (FAO, 2010, UICN, 2010) montrent que les ressources naturelles sont en perpétuelle dégradation dans les aires protégées, malgré les efforts consentis pour la conservation. Les principales causes de ces pressions sont essentiellement la pauvreté, l'agriculture extensive et la transhumance (Dimobe et al., 2012).

\footnotetext{
Universidad de Lomé (Togo); E-mail: dabitora2004@yahoo.fr; http https://orcid.org/0000-0002-7533-6356

Universidad de Lomé (Togo); E-mail: kpwala75@yahoo.fr; https://orcid.org/0000-0002-7533-6356

Universidad de Lomé (Togo); E-mail: semakpavi@gmail.com

Universidad de Lomé (Togo); E-mail: diwedigaba@gmail.com; https://orcid.org/0000-0002-5947-4038

* Universidad de Lomé (Togo); E-mail: batawilakomlan@gmail.com; https://orcid.org/0000-0002-5947-4038

* Universidad de Lomé (Togo); E-mail: koffi100@gmail.com; https://orcid.org/0000-0003-4290-8861
} 
Dans ces conditions, l'écotourisme, en dépit de certains impacts négatifs est reconnu comme moyen efficace de gestion durable des aires protégées (Meral et al., 2009 ; UICN, 2010). Les pays comme le Botswana, l'Afrique du Sud, la Namibie, le Kenya et la Tanzanie, ont su mettre en valeur leurs aires protégées par l'écotourisme, avec des résultats satisfaisants sur les plans conservation et développement local (Lapeyre, 2010 ; Leung, et al., 2019). Cependant, dans ces pays, les offres écotouristiques proposées aux visiteurs sont généralement orientées vers la découverte des ressources naturelles des aires protégées. Les autres potentialités, surtout culturelles, des communautés riveraines, étant généralement négligées dans ces offres.

En Afrique du Sud, par exemple, la raison première motivant l'arrivée des touristes étrangers, reste la découverte de ses paysages naturels et de sa faune. Ceux-ci s'inscrivent dans les safaris au sein des nombreux parcs nationaux et réserves naturelles (Folio, 2016), proposée par la plupart des guides écotouristiques de ces aires protégées. Folio (2016) relève que ces touristes qui arrivent pour découvrir la biodiversité, finissent par avoir un engouement pour la découverte des sites historiques ou culturels.

De toute évidence, la valorisation des potentialités historiques ou culturelles, doit être davantage considérée dans les circuits écotouristiques proposés dans les aires protégées. Ces potentialités historiques pouvant susciter la curiosité de certains touristes, contribueront ainsi à la valorisation et préservation des ressources naturelles de ces aires protégées.

En Afrique de l'Ouest, où les ressources fauniques sont difficilement observables dans la plupart des aires protégées (UICN, 2010 ; Polo-Akpisso et al, 2016, Koumantiga et al, 2018), les potentialités historico culturelles représentent un atout majeur pour la conservation de la biodiversité. Généralement peu connues dans les pays en développement comme le Togo, les potentialités historiques restent inexploitées dans le cadre de la gestion efficace des aires protégées ou de développement local (Koumantiga et al., 2012).

Le Togo est un pays de l'Afrique de l'Ouest, disposant d'un important système national d'aires protégées et peu valorisé par l'écotourisme en raison de la raréfaction de la diversité faunique. Le complexe Oti-Kéran-Mandouri (OKM) est l'un des plus importants de ce système national d'aires protégées. Dans ce complexe, les activités écotouristiques sont rares en raison de son état de dégradation qui ne favorise pas l'observation de la faune. Pourtant, il est entouré de groupes ethniques diversifiés possédant chacun des éléments historiques pouvant contribuer à l'enclenchement des activités écotouristiques.

La non valorisation écotouristique de ces potentialités historiques s'expliquent en partie par l'absence des données sur ces dernières. Ainsi, ce potentiel est méconnu, puisque rares sont des études qui sont menées dans ce sens. Pour une meilleure valorisation des aires protégées par l'écotourisme, leurs potentialités historiques doivent être clairement identifiées, analysées et géolocalisées.

Cette étude s’inscrit dans la contribution à la gestion durable des aires protégées par l'écotourisme en prenant le cas du complexe OKM. Elle étudie les potentialités écotouristiques liées à l'histoire pouvant être valorisés autours des aires protégées. Ensuite, elle porte une analyse sur les enjeux de mise en valeur de ces types de potentialités dans la gestion durable des ressources naturelles « in situ».

\section{Revue de la littérature}

\subsection{Problématique de valorisation écotouristique des potentialités historiques dans la gestion des aires protégées}

De nos jours, l'un des grands défis de la conservation des aires protégées dans les pays en développement tel que le Togo, est le manque d'un mécanisme de financement de gestion durable de ces écosystèmes. Ainsi, la capacité de l'Etat à financer la protection des aires protégées est faible. Face à ces difficultés de mobilisation de financement, il s'avère indispensable de faire recours à des stratégies alternatives parmi lesquelles se trouve promotion de l'écotourisme dans les aires protégées.

L'écotourisme représente une des rares formes de développement touristique qui, sous certaines conditions, peut favoriser la protection des zones naturelles grâce aux programmes de conservation qu'il suscite et qu'il peut financer (PNUE, 2002).

Dans les aires protégées des pays en développement, les activités écotouristiques sont essentiellement tournées vers les ressources biologiques notamment la faune et la flore. Dans celles de l'Afrique de l'ouest, l'UICN (2010) souligne que le tourisme de vision de la grande faune est largement dominant. Cependant, les études de cas montrent que la probabilité d'observation de cette faune dans le cadre des activités touristiques est généralement faible (Koumantiga et al., 2018). La faible observation de la faune dans ces aires protégées conduit à leur faible valorisation écotouristique. 
Par exemple, au Togo, dans les années 1980, les ressources fauniques et leurs habitats étaient bien conservées dans les aires protégées notamment dans celles du complexe OKM. Les activités touristiques étaient prospères avec l'arrivée des milliers de touristes par an. A partir des années 1990, les troubles sociopolitiques ont entrainé l'envahissement de ces écosystèmes par les riverains avec pour conséquences, la diminution de la faune et la destruction des habitats (Dimobe et al., 2012). Dès lors, les activités touristiques ont fortement baissé. Dans ce contexte, l'Organisation Mondiale du Tourisme (OMT) souligne l'importance de développer le tourisme lié aux visites des sites historiques, artistiques, archéologiques (WTO, 2014).

Le patrimoine historique constitue donc un potentiel devant être exploité au maximum dans la valorisation des aires protégées par l'écotourisme. Dhaher (2012), souligne que le regain d'intérêt pour les sites historiques est souvent orienté vers une perspective de développement touristique. Sinou (2005), relève qu'à une échelle internationale, les vestiges historiques deviennent pour les visiteurs occidentaux, en nombre croissant, des repères d'une histoire universelle.

Dans le cas des pays africains, l'exploitation des potentialités historiques autour des aires protégées à des fins écotouristiques serait un vrai atout pour la conservation et gestion durable de ces écosystèmes. Pour ce faire, des investigations scientifiques poussées doivent être menées, afin de proposer des offres attrayantes et durables pour l'épanouissement des communautés locales.

\subsection{Potentialités historiques et développement de l'écotourisme.}

Le développement de l'écotourisme sur un espace dépend premièrement de ses richesses ou potentialités écotouristiques. Ces potentialités sont diversifiées et incluent les potentialités naturelles et culturelles. Les potentialités naturelles constituent le plus souvent la principale attraction des visiteurs dans les aires protégées en Afrique (Koumantiga et al., 2018). Thapa (2012), souligne dans ses travaux que les principales attractions du parc national de Kafue en Zambie sont la faune et les commodités naturelles.

Quant aux potentialités culturelles au sein desquelles se retrouvent les potentialités écotouristiques historiques, elles sont souvent peu valorisées dans les offres écotouristiques des aires protégées africaines et plus particulièrement au Togo.

Les potentialités historiques sont considérées comme l'ensemble des attractions touristiques liées à l'histoire des communautés. Romero Macías \& Pérez Lopez (2010) estiment que le potentiel historique regroupent les valeurs qui possèdent tous les éléments ou manifestations d'attraction qui nous rapprochent de la connaissance des civilisations passées. Ces types de potentialités ont un grand rôle pour les communautés locales et requièrent de nos jours, l'attention des travaux scientifiques sur l'écotourisme. Ainsi, en Languedoc-Roussillon, Montargot \& Borg (2017) ont porté leur étude sur la mise en tourisme d'un site culturel labellisé au patrimoine mondial de l'humanité à partir du cas du pont historique du Gard. Au Brésil, les travaux de Fontan (2011) ont analysé l'exploitation touristique des sites historiques de Igarassu.

Les différents travaux s'accordent sur l'important rôle des sites historiques dans les offres aux visiteurs. Bremner \& Wikitera (2016), soulignent que le tourisme joue un rôle important pour les sociétés autochtones dans la mesure où il peut servir d'outil pour reconquérir un lieu, rétablir l'identité culturelle et, éventuellement, réécrire l'histoire. Nascimento Brandao (2014) estiment que l'utilisation de l'histoire comme attractions potentielles permettra le développement économique des communautés autochtones.

De nos jours, le tourisme est une activité populaire qui n'est plus seulement exercé par la classe des élites. Une grande partie des visiteurs est très prédisposée à visiter des lieux où les formes de vie et de travail du passé sont expliquées (Romero Macías, \& Pérez Lopez, 2010). Les sites historiques attirent les voyagistes car ils constituent un complément indispensable aux attractions naturelles. En outre, ces sites ont des curiosités et des histoires qui peuvent être transformées en attractions et en produits touristiques consommables, à condition qu'ils soient accessibles aux visites organisées (Roura, 2009).

Au regard des différents travaux, la nécessité de mettre en valeur les potentialités historiques dans le cadre de la promotion de l'écotourisme devient de plus en plus grandissante. Cependant, la mise en valeur de ces potentialités doit se reposer sur les critères de durabilité. En effet, l'écotourisme, s'il est mal planifié, peut conduire à la dégradation des ressources sur lesquels il se fonde. La plupart des activités touristiques dans les pays en développement exercent une pression sur les ressources environnementales à l'origine même de ces activités (Carter, 1991). Fontan (2011), relève dans ses travaux que l'exploitation touristique des sites historiques de Igarassu au Brésil constituent un facteur de dévalorisation et de dégradation de ce patrimoine. 
Denais (2007), souligne que si le tourisme en général représente une activité intéressante d'un point de vue économique, il n'en demeure pas moins que celui-ci peut avoir des conséquences indésirables sur l'environnement ainsi que sur les populations d'accueil. Dans le développement de l'écotourisme, il faudra concilier les attentes des visiteurs, le bien-être à long terme des collectivités d'accueil (Lindberg, 1991) ainsi que de la protection de la nature.

$\mathrm{Au}$ Togo tout comme dans certains pays africains, la problématique de la faible valorisation des aires protégées par l'écotourisme s'expliquent par le fait que les offres touristiques reposent plus sur les éléments de la biodiversité tels que la faune, la flore. Cependant la plupart des aires protégées sont sous une forte pression anthropique avec comme conséquences, la raréfaction des éléments de la biodiversité (Dimobe et al., 2012 ;Polo-Akpisso, 2019 ; Ouoba et al., 2019). Dans ce contexte, pour relever les niveaux de fréquentations et de satisfactions des touristes dans les aires protégées des pays de l'Afrique de l'ouest, il faut élargir les offres touristiques des zones protégées à d'autres potentialités telles celles liées à l'histoire qui retiennent l'attention des touristes. Des investigations scientifiques doivent être menées dans les zones des aires protégées pour permettre d'analyser ces types de potentialités en vue de leur valorisation dans les stratégies de conservation des aires protégées.

\section{Méthodologie}

\subsection{Brève présentation de la zone d'étude}

L'étude est conduite au Nord Togo dans les préfectures riveraines du complexe d'aires protégées Oti-Kéran-Mandouri. Ce complexe est adjacent aux préfectures de l'Oti, de Kpendjal de la région des savanes et celle de la Kéran de la région de la Kara (Figure 1).

Le complexe Oti-Kéran-Mandouri est l'ensemble formé par deux aires protégées que sont le parc national Oti-Kéran et la réserve de faune de l'Oti-Mandouri. Dans les années 1980, la superficie du complexe OKM faisait, 327390 ha. Suite aux troubles sociopolitiques qu'a connu le Togo dans les années 1990, les populations riveraines ont envahi ce complexe et engendré de grandes conséquences sur les habitats. Dans les années 2000, un processus de réhabilitation des aires protégées du pays a conduit à la révision des limites des aires protégées du complexe OKM. Ainsi, la superficie de complexe révisée est de 179000 ha.

Ce complexe présente une importance régionale de par sa situation géographique. En effet il est en relation avec le complexe WAP qui s'articule autour de deux (2) unités, centrées sur le Parc du W (Bénin, Burkina Faso, Niger) et l'ensemble constitué d'Arly-Pendjari (Burkina Faso-Bénin). Reconnu comme réserve de Biosphère par la commission MAB de l'Unesco en 2011, le complexe d'aires protégées Oti-Kéran-Mandouri est classé site Ramsar depuis 2007.

Sur le plan humain, les principales villes de la zone du complexe OKM sont : Kanté pour la préfecture de la Kéran, Mango pour la préfecture de l'Oti et Mandouri pour la préfecture de Kpendjal. Les principaux groupes ethniques des communautés riveraines sont : les Lamba, les Tamberman et les Gam Gam pour la préfecture de la Kéran, les Tchokossi, les Moba et les Gam Gam pour la préfecture de l'Oti, les Gourmantché et les Moba pour la préfecture de Kpendal (Gayibor, 1997)

La population de ces trois préfectures est de 439645 personnes (DGSCN, 2011). L'Agriculture et l'élevage constituent les principales activités des populations. En majorité ces peuples ont conservé une grande partie de leurs traditions malgré l'influence des religions modernes comme l'islam et le christianisme. Par ailleurs, Les groupes ethniques Lamba, Tchokossi et Moba sont connus dans l'histoire du pays en raison de leur résistance face aux forces coloniales allemandes (De Haan, 1993).

\subsection{Collecte de données}

La collecte des données s'est déroulée dans les trois préfectures riveraines du complexe OKM notamment préfecture de la Kéran, de l'Oti et de Kpendjal. Elle a été réalisée auprès des populations locales ainsi que des strcutures techniques publiques et privées impliquées dans la gestion des aires protégées et du tourisme dans la zone. Dans ces préfectures, les enquêtes ethnographique et socio culturelles (investigations sur les considérations sociaux et culturels de certains sites) ont été menées dans tous les cantons.

Au cours des enquêtes, les personnes ont été interrogées par interview individuel et au sein de focus group composés chacun de cinq (05) à dix (10) personnes en moyenne. Les choix de personnes enquêtées dans les différents cantons ont été fait de manière raisonnée. Pour ce faire un échantillonnage raisonné à deux niveaux a été adopté. Le premier niveau prend en compte $88,88 \%$ soit 32 cantons des cantons des 
trois préfectures riveraines du complexe, choisis suivant le critère adjacence au complexe. Au second niveau, l'enquête a pris en compte toute personne âgée d'au moins 40 ans, vivant dans la zone cible, sans distinction de sexe. Au total 223 personnes ont été enquêtées en 30 jours au cours de l'année 2017. Des observations directes sur le terrain ont complètés les enquêtes

Les données collectées au cours des enquêtes portaient sur les reliques coloniales, les sites historico culturels des différents groupes ethniques et les grottes ancestrales. Les enquêtes ont été couplées de visites de terrains qui ont permis de géolocaliser et mieux décrire les sites dentifiés.

Figure 1: localisation du complexe OKM au nord Togo

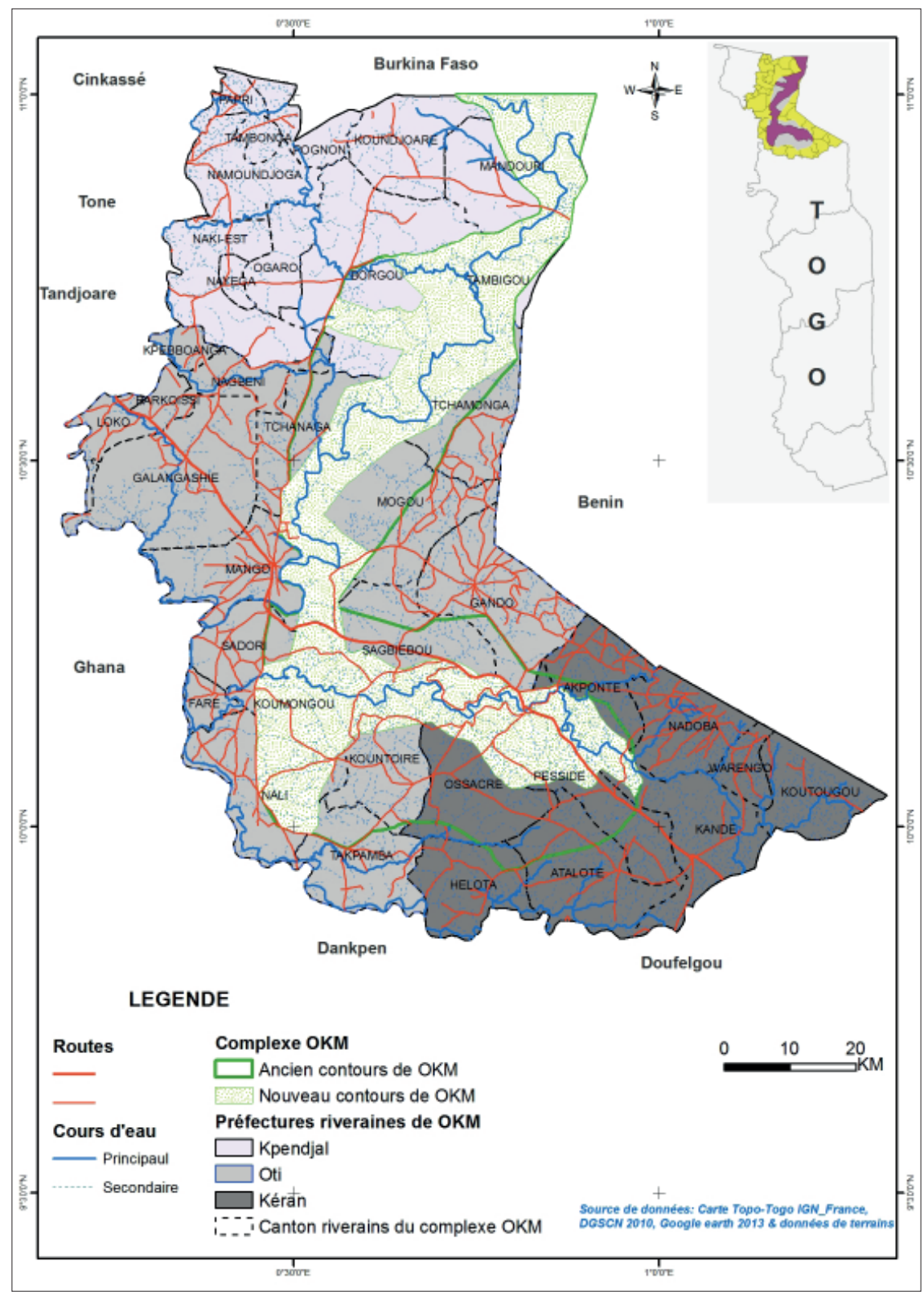




\section{Résultats}

Le complexe OKM et les localités riveraines disposent d'importantes potentialités écotouristiques historiques. Ces différentes potentialités traduisent l'histoire des groupes ethniques riverains du complexe, ou celle de toute la nation togolaise. Des investigations ont permis d'identifier un total de 52 sites historiques dans le complexe et ses localités riveraines. Ces sites se répartissent en trois catégories dont les grottes ancestrales au nombre de 14 sites soit $27 \%$ des sites, les reliques coloniales au nombre de 12 sites soit $23 \%$ des sites et les autres sites historiques au nombre de 26 sites soit $50 \%$ des sites. Suivant les différentes préfectures et le complexe OKM, on remarque que les sites de reliques coloniales sont bien représentés dans le complexe et dans la préfecture de l'Oti. Le nombre des principales grottes ancestrales est plus élevé dans la préfecture de l'Oti par rapport aux autres préfectures. Chacun des sites identifiés présente ses spécificités pouvant contribuer à sa valorisation par l'écotourisme dans la zone (Figure 2).

\section{Figure 2: Répartition des potentialités historiques suivant leur catégorie dans les différentes préfectures}

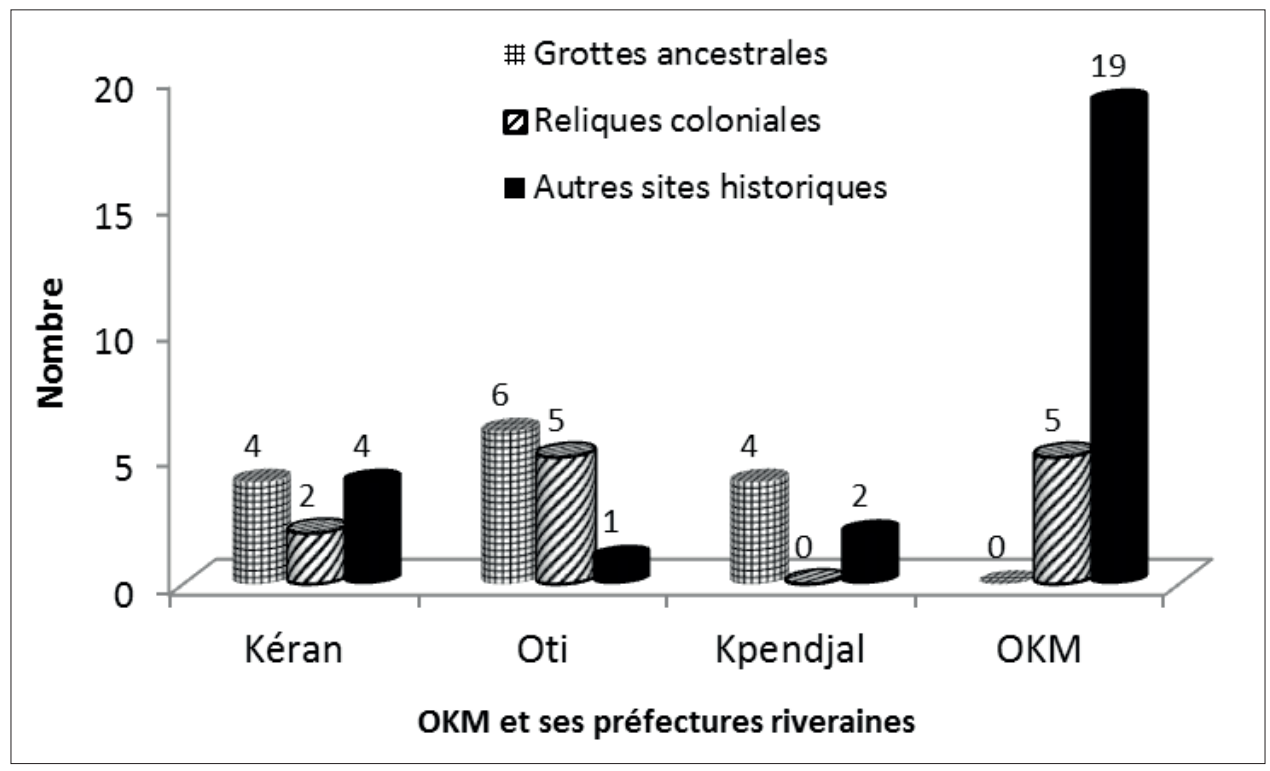

\subsection{Les grottes ancestrales}

L'histoire des communautés riveraines du complexe OKM est matérialisée par la présence des grottes ancestrales disséminées dans certaines localités. Pour la plupart de ces grottes, il s'agit des cavernes dans les pieds de baobab (Adansonia digitata) ou dans les collines qui jadis étaient les habitats des anciens hommes des différentes communautés riveraines du complexe OKM. Les grottes les plus spectaculaires sont :

Les grottes de Sogou dans les collines de Nayega, Sodjoal dans les monts de Naki-Est et celles de Nabonga située au cœur des collines plates de Namoudjoga. D'après les communautés, ces grottes constituent l'habitat des fondateurs de ces localités. Elles sont marquées par d'impressionnantes peintures rupestres. Il s'agit des peintures exécutées par ces hommes anciens hommes sur des grands blocs rocheux. Ces peintures rupestres représentent des formes humaines, animale, et des activités de ces hommes historiques. On y distingue par exemple, un cavalier sur son cheval, des représentations d'objets domestiques et artistiques de ces peuples. Au niveau de ces grottes, on peut observer des traces archéologiques marquées par des objets pierres taillées.

Ces peintures sont faites en couleur rouge sur les parois de la roche et sont bien visibles de jours dans ces grottes. (Photo 1). 
Photo 1: Grotte ancestrale à peintures rupestres de Namoudjoga

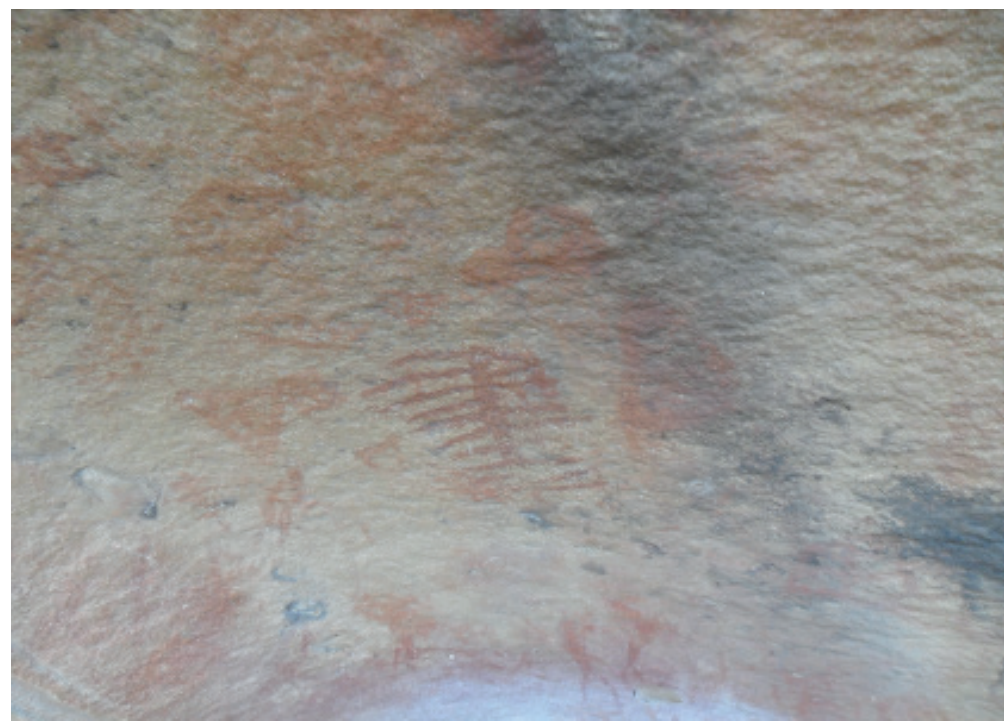

Auteur, 2017

La grotte de Bassamba dans le canton de Warengo est une grande cavité creusée à l'intérieur d'un gros pied de baobab (Adansonia digitata) faisant près de $14 \mathrm{~m}$ de circonférence. Elle s'ouvre à l'extérieur par une entrée soigneusement aménagée. Devant cette entrée se dressent des statuettes fabriquées avec des argiles (Photo 2). Dans la culture des peuples Tamberma, ces statuettes représentant les divinités qui veillent sur les membres qui habitent cette maison. A l'intérieur de la grotte on observe des escaliers en bois qui aboutissent à une ouverture vers le haut qui sert de fenêtre. D'après certains mythes cette grotte est la maison des premiers arrivés qui ont fondé le village de Bassamba. Ils y ont vécu durant des années, avant de commencer par construire les tatas Tamberma qui constituent l'habitat transitionnel des peuples Tamberma. Ces tatas, devenus patrimoine mondiale de l'UNESCO depuis 2004, ont toujours gardé certaines particularités de cette grotte notamment, l'escalier en bois, la fenêtre et les statuettes des divinités locales.

Photo 2: Grotte ancestrale Bassamba

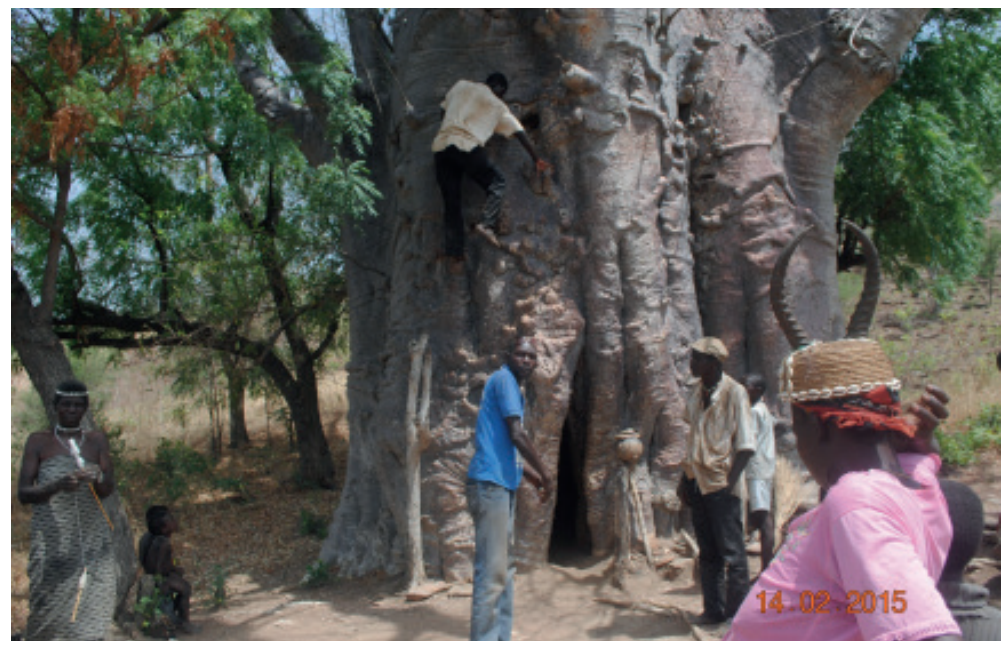

Auteur, 2015 
Les autres grottes spectaculaires se trouvent dans les cantons de Ossacré, Kountoiré, et Tchanaga.

Dans le canton de Ossacré on trouve les grottes " Ayikaworo " et " Nagbantelou ". Le premier nom désigne une grotte aux lions où vivait un ancêtre appelé « Ayikaworo ». Selon les mythes de la localité, cet ancêtre fut un guerrier tout puissant qui assurait la défense du village. Juste après sa mort, les lions représentant les divinités du village sont venus occuper cette grotte. C'est ces derniers qui protègent les populations de la localité contre les forces maléfiques. Le second désigne un baobab avec une caverne ancestrale ayant été habitée par les fondateurs du village de Ossacré.

La grotte " Bok " dans le canton de Kountoiré est une grande galerie où les grands parents, se réfugiaient en période de guerre. D'après les récits ils passaient près de 3 mois dans cette grotte et étaient nourris par un python géant. Cette grotte représente un des plus importants sites historiques du canton de Kountoiré et son histoire se transmet oralement de génération en génération.

Dans le canton de Tchanaga, la grotte " N'napoudouni " est reconnu sous le nom maman donne-moi de l'eau. D'après les mythes, cette grotte donnait de l'eau aux anciens hommes en période de sécheresse. Il suffit d'y aller et dire maman donne-moi de l'eau pour que l'eau commence par jaillir de la grotte.

\subsection{Reliques coloniales}

Les reliques coloniales regroupent essentiellement les ouvrages datant de la colonisation allemande au Togo, les pieds centenaires de fromager (Ceiba pentandra) plantés pendant l'époque coloniale et les cimetières des administrateurs coloniaux.

Les ouvrages coloniaux sont caractérisés par d'impressionnants ponts allemands, les ruines des bâtiments coloniaux, et les voies de ralliement des missionnaires coloniaux. Par rapport aux ponts coloniaux, cinq principaux ponts allemands ont été répertoriés. Ces ponts sont tous logés dans le complexe OKM. Le plus spectaculaire pont colonial, est le grand pont allemand érigé sur la rivière Koumongou qui traverse le complexe OKM.

\section{Photo 3: Paysage du tablier du grand pont allemand dans OKM}

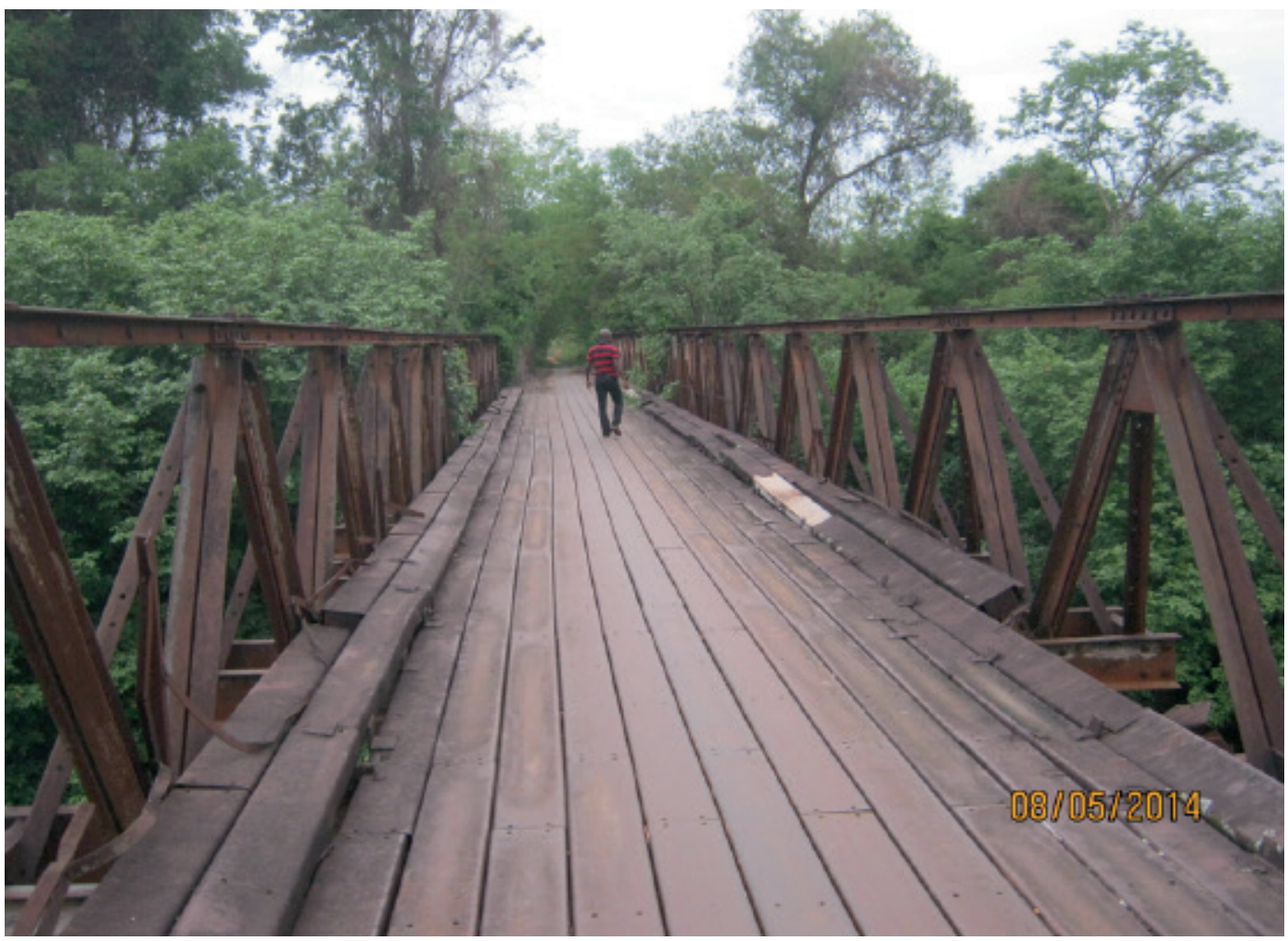

Auteur, 2014 
Ce grand pont allemand situé au cœur du parc Oti-Kéran est une œuvre magnifique et impressionnante. Il s'agit d'un grand pont à poutre construit manuellement par les grands parents des togolais au cours de la période coloniale allemande. Le tablier, partie du pont destinée à la circulation des véhicules est fait d'épaisses plaques métalliques. De part et d'autre du tablier, une rambarde constituée de grosses ferrailles solidement fixées sert de parapet. Le paysage de ce tablier et parapet au beau milieu des formations forestières denses demeurent très attractif (Photo 3). D'une portée d'environ $12 \mathrm{~m}$, ce pont de longueur totale près de $25 \mathrm{~m}$ repose sur une immense pile faite de béton. Ce béton est essentiellement constitué de blocs de cuirasses soigneusement taillés (Photo 4). Selon les révélations des enquêtés, ce grand ouvrage a été construit manuellement par leurs grands-parents sans aucun apport de machines ni de véhicules. Il traduit ainsi l'ampleur des travaux forcés de l'époque coloniale. Par ailleurs, la solidité et la vigueur de cet édifice datant de plus de 100 ans sont très impressionnantes. Au niveau de ce pont, la beauté du paysage des forêts galeries, la présence de divers gamme d'oiseaux et la présence des lianes et des multiples formes d'architectures racinaires retiennent l'attention.

\section{Photo 4: Pile du grand pont Allemand dans OKM}

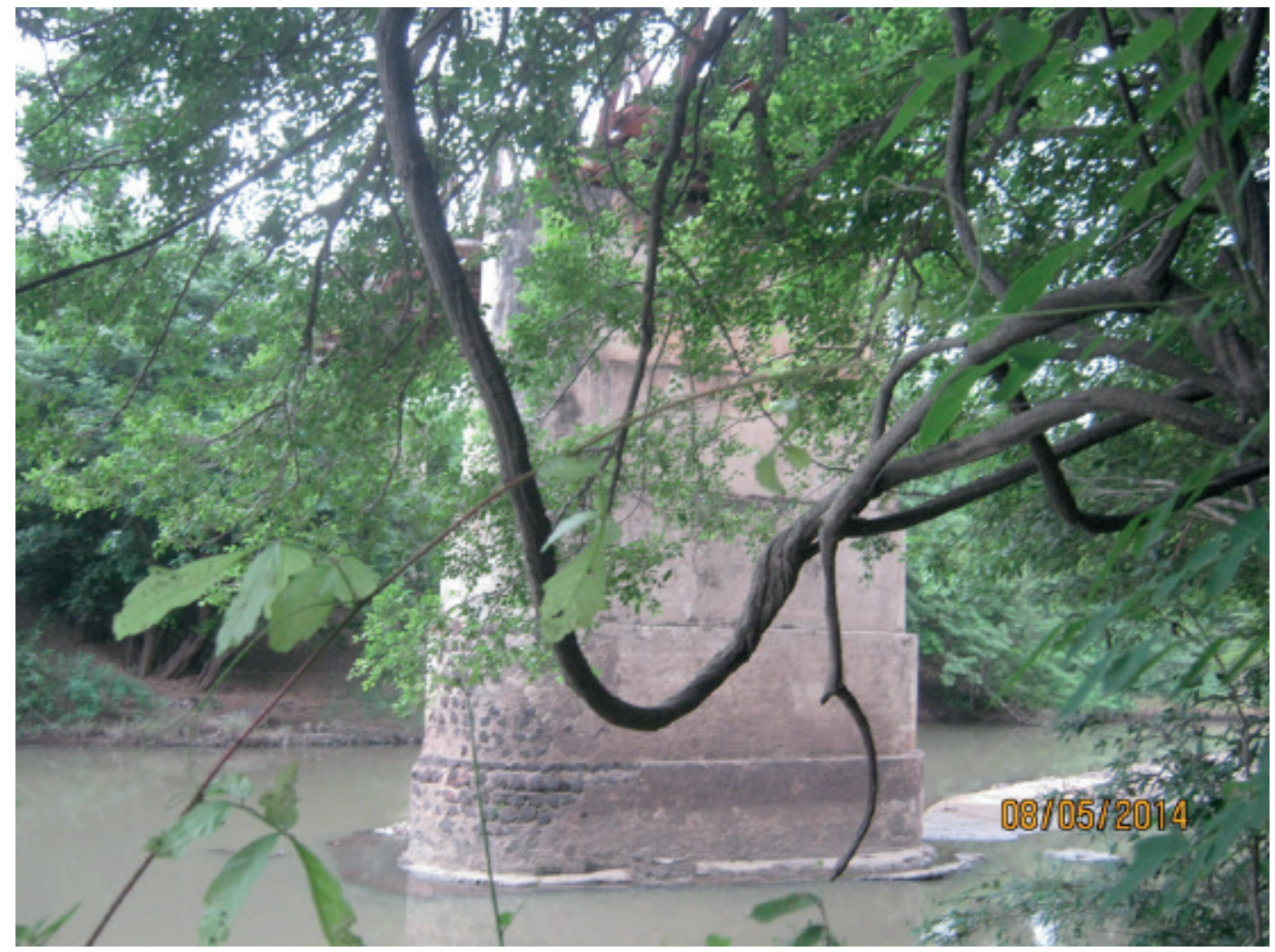

Auteur, 2014

Comme ouvrages coloniaux aussi remarquables, on relève les pistes coloniales. Ces dernières sont plus représentatives dans la préfecture de l'Oti. Il s'agit spécialement des routes des périples des missionnaires allemands de l'époque coloniale au nord Togo. Ces pistes sont en terres et caractérisées par la présence des arbres centenaires datant de l'époque coloniale allemande. La piste coloniale la plus remarquable dans la zone du complexe, quitte le canton de Nali jusqu'à la ville de Mango, en passant par les cantons de Faré, Koumongou et Sadori. Elle se distingue par la présence de pieds centenaires de fromager (Ceiba pentandra) qui la longent à plusieurs endroits. Ces pieds centenaires de fromager 
sont nettement remarquables avec leurs tailles et architectures. La hauteur moyenne des individus de ces pieds centenaires est de $28 \mathrm{~m}$ avec un diamètre moyen de $52 \mathrm{~cm}$. Ils présentent une architecture remarquable avec de jolis racines contreforts bien développées (Photo 5).

\section{Photo 5: Route coloniale bordée de pieds centenaires de fromager}

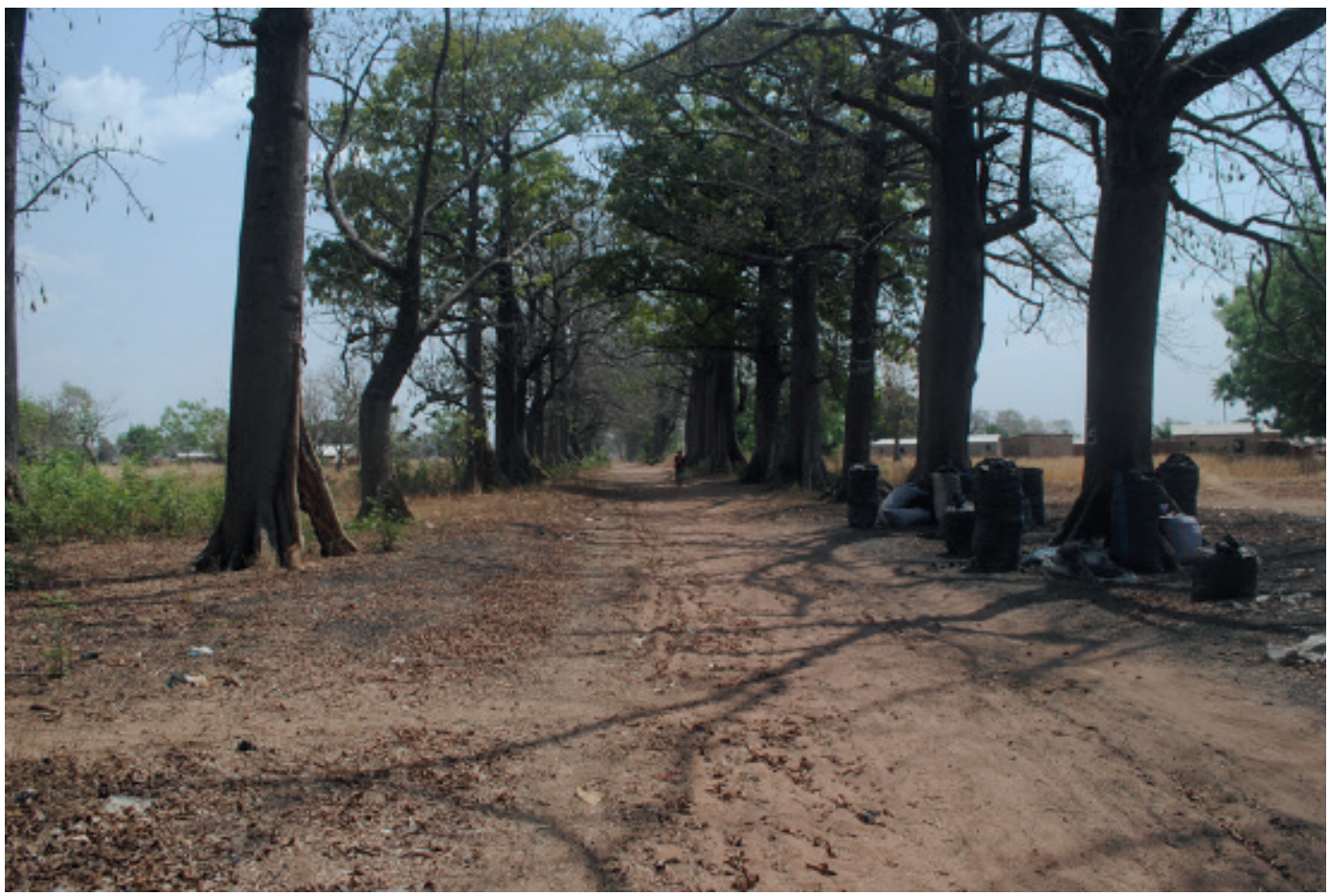

Photo Koumantiga, 2015

Les autres ouvrages coloniaux remarquables dans la zone du complexe regroupent le cimetière des colons, les bornes frontalières coloniales et les ruines des bâtiments coloniales. Le cimetière colonial localisé dans la ville de Mango est un site historique important pour la promotion de l'écotourisme. . Il comprend trois tombes des Allemands dont deux (2) étaient morts bien avant la guerre : Franz Werner (9 mars 1900), E. Heitmann (Décembre 1896) et Ferdinand dont l'équipage n'a pas pu être complètement restauré. Ce cimetière a été réaménagé en 1999 lors de la célébration de la journée du deuil commémorant les batailles des alliés Français et Anglais contre les Allemands pendant la première guerre mondiale.

En ce concerne, les bornes frontalières, elles se trouvent dans le canton de Nadoba vers l'Est. Ces bornes sont implantées pendant la période de tutelle française pour déterminer la frontière le Togo et le Dahomey (actuelle république du Bénin). Enfin, les vestiges coloniaux marqués par les ruines des batiments coloniaux sont encore conservés dans le canton d'Ataloutè. Ces bâtiments datant de l'époque coloniale allemande étaient construits pour loger les missionnaires Allemands.

\subsection{Autres sites historiques liés à l'histoire des peuples}

Dans toutes les communautés, on retrouve une diversité de sites qui se rapportent à l'histoire de la communauté. Il s'agit des lieux que les populations locales gardent de génération en génération. Des mythes relatifs à ces sites sont bien connus et racontés aux étrangers. Parmi ces sites historiques on peut relever la présence de dix-neuf (19) sites fétiches mentionnés dans le complexe OKM. Ces sites font partie de l'histoire des peuples riverains de ce complexe et les personnes âgées peuvent la raconter. 
Il s'agit des sites correspondant généralement à des vieux pieds d'espèces végétales, soit à un ilot de forêts ou encore à un endroit précis d'un cours d'eau. Les communautés riveraines sont liées à ces sites où elles doivent aller annuellement faire des sacrifices qui débouchent souvent sur des manifestations culturelles. On peut relever comme exemple, sept (7) sites correspondant à de vieux individus de Adansonia digitata sous lesquels se font des sacrifices chaque année. Une forêt claire où des jarres enterrées, représentant la divinité locale est également relevée. Aussi, a-t-il été signalé quatre (4) sites où se font des sacrifices au niveau des cours d'eau Koumongou et Oti.

De nos jours, la localisation de ces sites dans le complexe OKM est mal connue par les jeunes et plus de $75 \%$ des répondants reconnaissent que la fréquence des sacrifices au niveau de ces sites a fortement diminué. Les raisons relevées pour expliquer cet abandon sont la crainte de la répression des agents forestiers, les difficultés économiques de réunir les moyens nécessaires pour les sacrifices et la faible motivation des nouvelles générations liées aux nouvelles croyances.

Les autres sites historiques se retrouvent dans les différentes localités de la zone.

Dans le canton de Nayega, on note trois sites notamment, les trente cases rondes de Nayega et le site "Natorgou ". Les trente cases rondes de Nayega est un lieu historique où trente cases de formes circulaires avaient été construites par les anciens pour isoler les personnes atteintes de maladies contagieuses. Selon les enquêtés ces cases construites traditionnellement, datent de plus de 200 ans et certaines ne sont pas encore totalement détruites. Le site "Natorgou " est un lieu où on observe un pied de cheval bien moulé sur un grand rocher. Ce site démontre la puissance que les ancêtres guerriers possédaient pour vaincre l'ennemi et fait partie de l'histoire des natifs du canton de Nayega.

Dans le canton d'Atalouté, on retrouve site "Afalowéré » où se pratique la lutte des jeunes. Cette lutte consiste à soulever de façon haltérophilique un rocher historique d'environ $50 \mathrm{~kg}$. Ce rocher historique est soigneusement conservé sur ce site et le jeune qui parvient à soulever démontre sa puissance.

Dans le canton de Nadoba on relève deux sites historiques: les sites "Likpo " et "Katamatchiata». Le site «Likpo » est une forêt sacrée située où se déroule l'initiation des jeunes Tamberma. Le site «Katamatchiata " est une maison historique où commence la cérémonie d'initiation des jeunes Tamberma du clan Batamaba. Après la cérémonie, les jeunes initiés pratiquent la danse du fouet.

Enfin dans le canton de Kountoiré, on retrouve un site marqué par une pièrre sacrée. Cette pierre sacrée qui alimente l'histoire du canton vient de la transformation de l'un des ancêtres fondateurs du village du canton.

\subsection{Distribution spatiale des potentialités écotouristiques historique de la zone de OKM}

La répartition spatiale des différents sites historiques montre que ces potentialités historiques sont disséminées sur toute l'étendue de la zone. Cependant, certaines spécificités se dégagent. L'extrême nord caractérisé par la culture des peuples Gourmatché est un grand pôle de potentialités historiques caractérisées par une grande représentativité des grottes ancestrales. Du coté extrême Sud Est on retrouve aussi une zone particulière marquée par la culture des Tamberma et une diversité de sites historiques. (Figure 3). 
Figure 3: Distribution spatiale des potentialités écotouristiques historiques

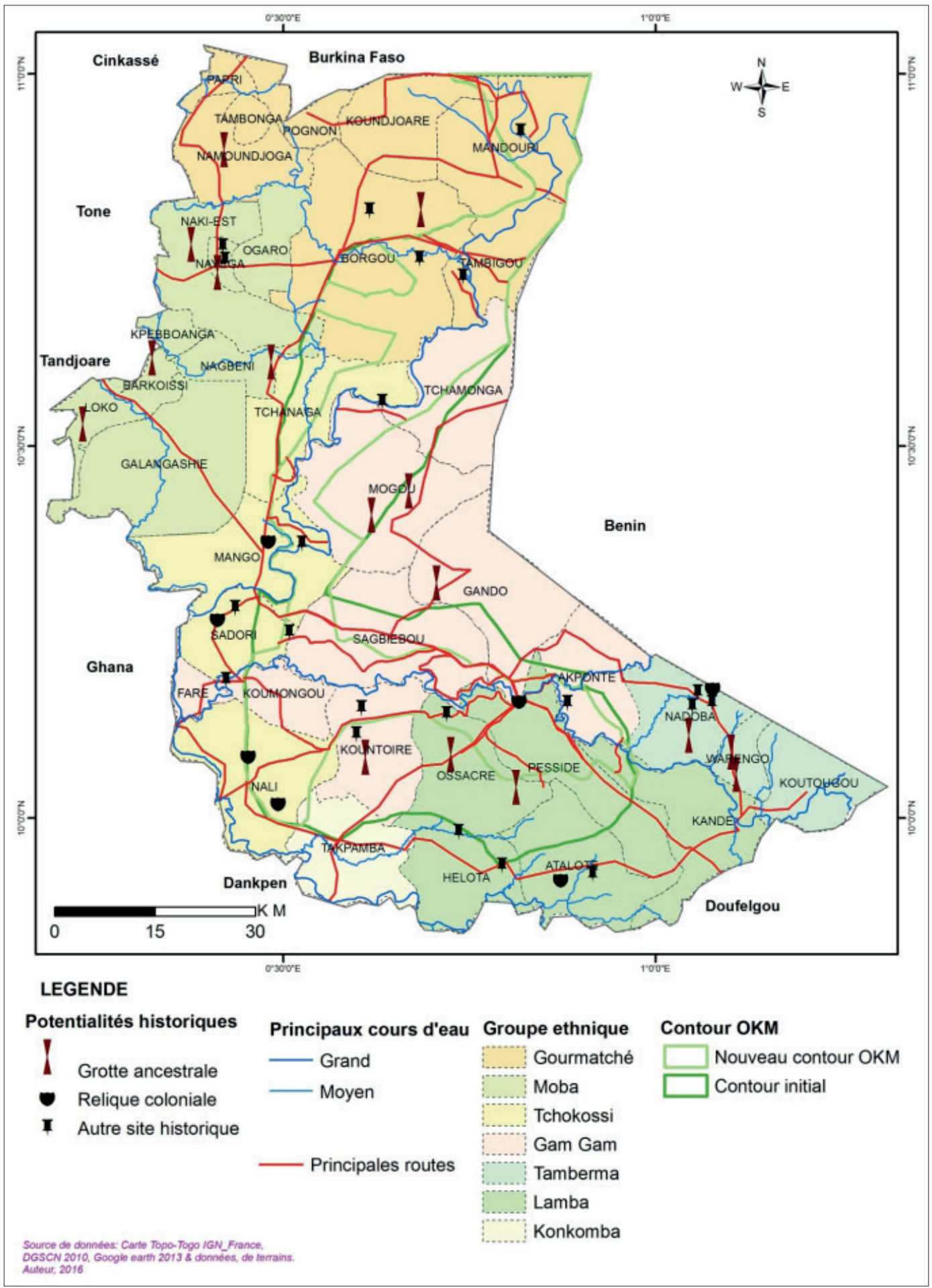




\section{Discussion: Enjeux de mise en valeur du potentiel historique dans les zones des aires protégées}

Considérer uniquement, la biodiversité dans les offres écotouristiques des aires protégées, expliquerait en partie les causes des dysfonctionnements dans le domaine de l'écotourisme dans certaines aires protégées de l'Afrique. En effet, plusieurs travaux relèvent que les aires protégées africaines sont diversement menacées par les activités anthropiques (Diallo et al.; 2011 ; Inoussa et al, 2011 ; Dimobe et al, 2012 ; Wala et al, 2012). Ces pressions engendrent une raréfaction des ressources fauniques qui sont difficilement observables sur les circuits touristiques (Koumantiga et al., 2018). Ceci affecte négativement l'arrivée des touristes dans ces écosystèmes car ces derniers ne sont pas satisfaits par rapport à leurs attentes de l'offre proposées.

Les grottes ancestrales disponibles dans les différentes localités riveraines de OKM constituent une opportunité pour proposer des produits touristiques orientés sur la culture historique. Les vestiges coloniaux notamment, ouvrages coloniaux, les cimetières des missionnaires coloniaux, sont de vrais mobiles pour attirer les nombreux touristes occidentaux en quête de la culture historique. Ces potentialités historiques peuvent être mises en valeurs par le tourisme culturel, mémoriel ou noir. Ces formes de tourisme sont en évolution et bien illustrées dans la Sénégambie (Bassène, 2011 ; Baillargeon, 2016). Dans la république Cap-verdienne, les traces coloniales se patrimonialisent dans une optique de valorisation touristique (Lesourd, 2011)

Aujourd'hui, alors que le développement du tourisme prend des dimensions notables, la réflexion sur la patrimonialisation et la préservation des sites historiques est devenue d'actualité, voire nécessaire (Dhaher, 2012). Les grottes à peintures rupestres de Namoudjoga, et de Nayega regorgent des objets archéologiques notamment des objets lithiques et en silex qui n'ont pas fait objet de datation. Cependant, ces types d'objets prouvent qu'il s'agit des sites préhistoriques qui méritent d'être entretenus. Des recherches archéologiques et autres plus poussées permettront d'approfondir les connaissances de ces sites et faciliter leur proposition pour inscription sur la liste du patrimoine mondial. Il incombe à chaque pays de désigner des sites du patrimoine situés sur son territoire en vue de leur inscription sur la liste du patrimoine mondial (Ratz \& Puczko, 2003).

Cependant, il faut relever que les débats sur les sites inscrits au patrimoine mondial sont larges et partagés. En effet, le tourisme peut avoir des effets à la fois bénéfiques et dommageables sur le patrimoine naturel et culturel (Ratz \& Puczko, 2003). La valorisation touristique d'un site du patrimoine mondiale augmente son attractivité et en même temps, risque de le détruire. Cependant, il est reconnu qu'à long terme, la protection d'un site classé sur la liste du patrimoine mondial pourrait avoir des impacts positifs sur les dimensions économiques, environnementales et sociales (Marcotte \& Bourdeau, 2010).

De nos jours la reprise de la coopération allemande se renforce en Afrique dans plusieurs domaines. Ceci constitue une opportunité pour le complexe OKM de mettre en exergue les diverses potentialités historiques surtout celles qui se rapportent à la colonisation allemande. C'est un avantage pour ce complexe de disposer en son sein des potentialités biologiques et des sites historico culturels. Des études ont relevé qu'un site naturel qui recèle des biens biologiques, culturels et historique est un pôle où les activités touristiques peuvent prendre de l'ampleur (Paniazza \& Piacente, 2008 ; Pralong, 2004). La présence des sites liés à l'histoire des communautés riveraines dans les aires protégées du complexe OKM, témoignent de l'enjeu culturel des aires protégées. Ainsi, on ne peut pas imaginer une stratégie de conservation et de et gestion efficace des aires protégées, sans la mettre en relation avec la culture des communautés riveraines. Dans ce contexte, l'écotourisme sous toutes ses dimensions est préconisée dans les zones des aires protégées.

Par ailleurs, Les œuvres allemandes décrites dans le complexe rappellent les facettes de la colonisation des pays africains. Dans ce contexte, le tourisme mémoriel et le tourisme sombre peuvent s'opérer autour de ces curiosités historiques avec une large implication des visiteurs nationaux et internationaux. En Pologne, le tourisme mémoriel fait drainer près de 1,4 millions de visiteurs chaque année sur le site d'Auschwitz (Crahay, 2015). Au Bénin, ce sont les sites précoloniaux du triangle historico-culturel d'Abomey, Ouidah et Porto-Novo, qui alimentent le tourisme international (Principaud, 2004).

Partant du cas du complexe OKM, on constate que les aires protégées et leurs localités riveraines disposent d'un patrimoine historique généralement peu exploité dans le cadre de la promotion de l'écotourisme. Cependant, la valorisation de ces potentialités historiques, par l'écotourisme, pourra avoir des effets positifs sur la conservation des ressources naturelles dans ces écosystèmes. Cette forme de tourisme couplée à l'éducation pourrait engendrer l'épanouissement des touristes et des populations locales qui cerneront mieux l'utilité de préserver les ressources naturelles. Dans ses travaux portant 
sur le lien entre l'éducation environnementale et l'écotourisme, de Souza Barros (2014) souligne que l'éducation environnementale devient un outil essentiel pour la mise en œuvre d'un tourisme répondant aux besoins des piliers du développement durable.

Plusieurs travaux ont montré l'impact positif de l'écotourisme sur les plans socioéconomiques et conservation des ressources naturelles (Clergeau, \& Etcheverria, 2013 ; Blondy, 2016 ; Yomb \& Tefe (2016). Ainsi, la valorisation de ces sites historiques par l'écotourisme va contribuer non seulement à la sauvegarde de ces sites pour les générations futures, elle va de même contribuer à la diversification des activités économiques dans les localités riveraines des aires protégées. Dans ce contexte la pression humaines sur les aires protégées va diminuer car, les ressources fauniques et floristiques ne seront pas les seules sources de revenus dans ces milieux ruraux.

Toutefois, il convient de relever que certains travaux ont montré que l'écotourisme générait des impacts négatifs sur la conservation des ressources naturelles (Andrianambinina \& Froger, 2006). Tardif (2003), estime que malgré les doutes et les remises en questions qui secouent aujourd'hui ce domaine de recherche, l'écotourisme demeure une piste riche à explorer afin de concilier le développement socioéconomique de nos sociétés et la protection de l'environnement.

Pour promouvoir l'écotourisme dans les aires protégées à l'instar de celles du complexe OKM, les sites historiques constituent un grand potentiel à exploiter. Dans ce contexte, l'état devra jouer son rôle par l'adoption des mesures incitatives pour encourager la mise en valeur de ces potentialités. Pour montrer le rôle de l'Etat, dans la promotion du tourisme, Meira Martoni \& Tarlombani da Silveira (2014) soulignent que pouvoir exercer par voie de la structure de l'Etat peut conduire à une certaine autonomie opérationnelle.

\section{Conclusion}

L'étude des potentialités écotouristiques liées à l'histoire de la zone complexe OKM au Togo a permis d'identifier 52 sites qu'on peut regrouper en trois catégories. Ainsi, on distingue les grottes ancestrales, les reliques coloniaux et les autres sites historiques liés à l'histoire des localités riveraines au complexe. Ce patrimoine historique qui devrait être exploité au maximum dans le cadre de valorisation des aires protégées par l'écotourisme reste un potentiel latent dans la plupart des pays en développement. Malgré les dimensions historico culturelles de l'écotourisme, les activités écotouristiques restent focalisées sur les potentialités biologiques dans les zones des aires protégées. Au regard de la dégradation des ressources biologiques dans les aires protégées ouest africaines, l'écotourisme doit prendre en compte les potentialités historiques, et culturelles. Ces dernières significatives dans les zones des aires protégées renforceraient les offres touristiques avec des impacts positifs sur les principes de conservation des ressources naturelles des aires protégées. Dans le cadre de développement des stratégies de gestion durable et efficace des ressources naturelles des aires protégées, il revient aux chercheurs de mener des investigations pour contribuer à la connaissance des autres potentialités écotouristiques dans les zones des aires protégées.

\section{Bibliographie}

Andrianambinina, D. \& Froger, G. 2006. «L'écotourisme, facteur de développement durable dans un contexte de mondialisation? Le cas de Madagascar». La mondialisation contre le développement durable, 281-310.

Bassène P. C. B. 2011. "Mémoire de l'esclavage et de la traite négrière en Sénégambie (1965-2007) Dialectique de la diversité mémorielle». Thèse de doctorat en cotutelle présentée à la Faculté des études supérieures de l'Université Laval, Québec, 3

Baillargeon T. 2016. «Le tourisme noir: l'étrange cas du Dr Jekyll et de M. Hyde». Téoros. Revue de recherche en tourisme, $35(35,1)$.

Blondy C. 2016. «Le tourisme, un facteur de développement durable des territoires insulaires tropicaux? Tourisme, aménagement, environnement et société locale à Bora Bora (Polynésie française)». Mondes du Tourisme, (Hors-série).

Bremner, H. \& Wikitera, K. A. 2016. «Using history for tourism or using tourism for history? Examples from Aotearoa/New Zealand». Journal of Tourism History, 8(3), 260-274. 
Busquet, M. B. 2006. «Des stratégies intégrées durables: savoir écologique traditionnel et gestion adaptative des espaces et des ressources». VertigO-la revue électronique en sciences de l'environnement, 7(2).

Cater, E. 1991. «Sustainable tourism in the Third World: problems and prospects». Sustainable tourism in the Third World: problems and prospects, (3).

Clergeau, C. \& Etcheverria, O. 2013. «La mise en tourisme et le développement local par la création d'une atmosphère gastronomique. Analyse à partir du cas de Vonnas». Mondes du Tourisme, (7), 52-67.

Crahay, F. 2014. «Tourisme mémoriel. Témoigner. Entre histoire et mémoire». Revue pluridisciplinaire de la Fondation Auschwitz, (117), 151-152.

De Haan, L. 1993. «La région des Savanes au Togo: l'Etat, les paysans et l'intégration régionale, 1885-1985». Karthala Editions.

Denais, L. 2007. «Écotourisme, un outil de gestion des écosystèmes» octoral dissertation, Université de Sherbrooke.

DGSCN. 2011. «Recensement général de la population et de l'habitat (RGPH4); Rapport final. DGSCN, République du Togo».65p.

de Souza Barros, J. D. 2014. «Educação ambiental no ecoturismo: potencialidades e estratégias de conservação dos recursos naturais». Revista Brasileira de Educação Ambiental (RevBEA), 8(1), 42-49.

Dhaher, N. 2012. «Les ambivalences de la mise en tourisme du patrimoine. Le cas du centre ancien de Tozeur (Tunisie)». Mondes du Tourisme, (6), 23-33.

Diallo, H., Bamba, I., Barima, Y. S. S., Visser, M., Ballo, A., Mama, A. \& Bogaert, J. (2011). «Effets combinés du climat et des pressions anthropiques sur la dynamique évolutive de la végétation d'une zone protégée du Mali (Réserve de Fina, Boucle du Baoulé)». Science et changements planétaires / Sécheresse, 22(2), 97-107.

Dimobe, K., Wala, K., Batawila, K., Dourma, M., Woegan, Y. A., \& Akpagana, K. 2012. «Analyse spatiale des différentes formes de pressions anthropiques dans la réserve de faune de l'Oti-Mandouri (Togo)». VertigO-la revue électronique en sciences de l'environnement, (Hors-série 14).

FAO. 2010. «Evaluation des ressources forestières mondiales 2010». Rapport principal. Etude Fao forêts $\mathrm{N}^{\circ} 168$. Rome, 377p.

Folio, F. 2016. «Dark tourism ou tourisme mémoriel symbolique? Les ressorts d'un succès en terre arc-en-ciel». Téoros. Revue de recherche en tourisme, 35(35, 1).

Köhler, A. F. 2011. «Patrimônio cultural, turismo e gestão pública: exploração turística predatória e desvalorização patrimonial em Igarassu, Brasil». Pasos: Revista de Turismo y Patrimonio Cultural, 9(2), 265-278.

Gayibor, N.L. 1997. «Histoire des togolais, des origines à 1884». Presses UB, Lomé, vol. 1:13-68.

Inoussa, M. M., Mahamane, A., Mbow, C., Saadou, M., \& Yvonne, B. 2011. «Dynamique spatio-temporelle des forêts claires dans le Parc national du W du Niger (Afrique de l'Ouest)». Science et changements planétaires/Sécheresse, 22(2), 108-116.

Koumantiga, D., Wala, K., Batawila, K., Akpagana, K. 2012 «Potentialités écotouristiques dans la préfecture de Doufelfgou». Etudes caribéennes, (23)

Koumantiga, D., Wala, K., Kanda, M., Dourma, M., \& Akpagana, K. 2018. «Aires protégées et écotourisme de vision de la grande faune: développement d'une approche méthodologique pour évaluer les circuits et application au complexe Oti-Kéran-Mandouri au Togo (Afrique de l'Ouest)». Études caribéennes, (41).

Lapeyre, R.

2010. " Le tourisme communautaire comme un moteur de développement durable en Namibie?". Tourisme durable dans les Suds?, 11, 193.

Lesourd M. 2011. "«Traces coloniales.» Le Blanc» et» l'indigène», regards-traces croisés dans la mondialisation». L'homme trace, Paris, Éditions du CNRS.

Leung, Y., Spenceley, A., Hvenegaard, G. \& Buckley, R. 2019. «Gestion du tourisme et des visiteurs dans les aires protégées : Lignes directrices pour la durabilité. Lignes directrices des meilleures pratiques dans les aires protégées No. 27, Gland, Suisse :UICN. 137p.

Mama, A., Sinsin, B., De Cannière, C. \& Bogaert, J. 2013. «Anthropisation et dynamique des paysages en zone soudanienne au nord du Bénin». Tropicultura, 31(1), 78-88.

Lindberg, K. 1991. "Policies for maximizing nature tourism's ecological and economic benefits». (pp. 20-21). Washington, DC: World Resources Institute.

Marcotte, P. \& Bourdeau, L. 2010. «La promotion des sites du Patrimoine mondial de l`UNESCO: Compatible avec le développement durable?». Management \& avenir, (4), 270-288. 
Meira Martoni, R., \& Tarlombani da Silveira, M. A. 2014. «Perspectives analytiques à considérer à propos du rôle de l'Etat dans le tourisme». PASOS. Revista de Turismo y Patrimonio Cultural, 12(4).

Méral, P., Froger, G., Andriamahefazafy, F., \& Rabearisoa, A. 2009. «Le financement des aires protégées à Madagascar: de nouvelles modalités». Aires protégées, espaces durables, 135-155.

Montargot, N. \& Borg, X. 2017. «Gestion touristique de sites culturels et relations entre parties prenantes: Le cas du pont du Gard». Téoros. Revue de recherche en tourisme, 36(36, 1).

Morais, D. B., Lee, S. H., Hou, J. S., Lin, C. H., Yarnal, C. M., \& Chick, G. 2010. «Heritage Tourism in Taiwan's Desinicized Nationalism»: Pasos: Revista de Turismo y Patrimonio Cultural, 8(2), 277-292.

Nascimento Brandão, C., Barbieri, J. C., Reyes Junior, E. \& de Moura João, C. 2014. «Análise da produção científica internacional sobre turismo indígena de 1990 a 2013: um estudo bibliométrico e proposição de uma agenda de pesquisa». Pasos. Revista de Turismo y Patrimonio Cultural, 12(4).

Ouoba, P; Yaméogo, T.J. \& Boussim, I. 2019. «Potentiel Écotouristique des Ressources Végétales de la Réserve Naturelle de Niangoloko, Sud-ouest du Burkina Faso». European Scientific Journal 15(9):22.

Panizza, M. \& Piacente, S. 2008. "Geomorphosites and geotourism». Rev. Geogr. Acadêmica, 2(1), 5-9.

PNUE. 2002. «L'avenir de l'environnement mondial». Rapport-Synthese, Geo3, Nairobi, 20 p.

Pralong, J. 2004. «Pour une mise en valeur touristique et culturelle des patrimoines de l'espace Alpin : le concept d'histoire totale». Histoire des Alpes 9, 301-310.

Principaud, P. 2004. «Le tourisme international au Bénin : une activité en pleine expansion», Les Cahiers d'Outre-Mer com.revues.org/529; DOI : 10.4000/com.529

Polo-Akpisso, A., Wala, K., Ouattara, S., Foléga, F., \& Tano, Y. 2016. «Changes in Land Cover Categories within Oti-Kéran-Mandouri (OKM) Complex in Togo (West Africa) between 1987 and 2013». In Implementing Climate Change Adaptation in Cities and Communities (pp. 3-21). Springer International Publishing.

Polo-Akpisso, A., Wala, K., Soulemane, O., Foléga, F., Akpagana, K., \& Tano, Y. 2019. «Assessment of Habitat Change Processes within theOti-Keran-Mandouri Network of Protected Areas in Togo (West Africa) from 1987 to 2013 Using Decision Tree Analysis”. Sci, 1(1), 9.

Ratz, T. \& Puczko, L. 2003. "A world heritage industry?: tourism at Hungarian World Heritage sites= Une industrie patrimoniale mondiale? le tourisme dans les sites du patrimoine mondial en Hongrie». In Regards croisés sur le patrimoine dans le monde à l'aube du XXIe siècle (pp. 467-481).

Romero Macías, E. \& Pérez López, J. M. 2010. «El Puerto de La Laja (Huelva), como Bien de Interés Cultural en la categoría de Sitio Histórico». Pasos. Revista de Turismo y Patrimonio Cultural, 8(4), 643-652.

Roura, R. 2009. «The polar cultural heritage as a tourism attraction: a case study of the airship mooring mast at Ny-Ålesund, Svalbard». Téoros: Revue de recherche en tourisme, 28(1), 29-38.

Sinou, A. 2005. «Enjeux culturels et politiques de la mise en patrimoine des espaces coloniaux». Autrepart, (1), 13-31.

Tardif, J. 2003. «Écotourisme et développement durable». VertigO-la revue électronique en sciences de l'environnement, 4(1).

Thapa, B. 2012. «Why did they not visit? Examining structural constraints to visit Kafue National Park, Zambia». Journal of Ecotourism, 11(1), 74-83.

UICN. 2010. «Le tourisme dans les aires protégées d'Afrique de l'ouest: Quelle contribution à la conservation?» Rapport étude PACO N 6. Ouagadougou, BF: UICN/PACO, 110p.

UNESCO. 2004. "World Heritage Scanned Nomination; Koutammakou, the Land of the Batammariba». Excerpt from the Report of the $28^{\text {th }}$ Session of the World Heritage Committee. 267p.

WTO. 2014. "Annual Report 2013, UNWTO» Madrid, Spain, 45p.

Wala, K., Woegan, A. Y., Borozi, W., Dourma, M., Atato, A., Batawila, K. et Akpagana, K. 2012. «Assessment of vegetation structure and human impacts in the protected area of Alédjo (Togo)». African Journal of Ecology, 50, 355-366

Yomb, J. et Tefe, T. R.2016. «Tourisme et socio-économie dans les espaces ruraux au Cameroun : entre survivances culturelles et mutations». Etudes caribéennes. revues.org/8820

Recibido:

$22 / 05 / 2018$

Reenviado:

$07 / 01 / 2019$

Aceptado:

$17 / 07 / 2020$

Sometido a evaluación por pares anónimos 